\title{
DECONVOLVING THE BEAM IN SMALL ANGULAR SCALE CMB EXPERIMENTS
}

\author{
J.V. Arnau ${ }^{1}$ and D. Sáez ${ }^{2}$ \\ ${ }^{1}$ Departamento de Matemática Aplicada, Universidad de Valencia. \\ 46100 Burjassot, Valencia, Spain \\ ${ }^{2}$ Departamento de Astronomía y Astrofísica, Universidad de Valencia. \\ 46100 Burjassot, Valencia, Spain \\ e-mail: jose.arnau@uv.es; diego.saez@uv.es
}

\begin{abstract}
This paper is concerned with experiments which measure CMB anisotropies on small angular scales. A certain coverage, a beam structure and a level of uncorrelated noise define each experiment. We focus our atention on the reversion of the beam average. In each experiment, we look for the best pixelization for reversion, namely, for the pixelization that -after reversion- leads to good maps containing right spectra for the most wide range of angular scales. Squared pixels having different sizes "smaller" than the beam radius $\left(\theta_{F W H M}\right)$ are considered. For a given size, the following question arises: How well can we assign a temperature to each pixel? Various mathematical methods are used to show that, in practice, this assignation -beam reversion or deconvolution- only leads to right spectra for pixel sizes greater than a certain lower limit close to
\end{abstract}


$\theta_{F W H M} / 2$. This limit is estimated for negligible and relevant levels of noise and also for spherically symmetric and asymmetric beams. After this general study, we focus our attention on two feasible detectors (which have been proposed to be on board of PLANCK satellite). For each of them, we estimate the size of the most appropriate pixelization compatible with beam reversion, difraction, observational strategy et cetera and, then, we answer the following question: Which is the part of the angular power spectrum which can be extracted from appropriately pixelized maps after deconvolution?

Subject headings: cosmic microwave background - cosmology:theory — large-scale structure of the universe - methods:numerical

PACS: 98.70.Vc, 95.75.-z, 95.75.pq, 95.75.St 


\section{INTRODUCTION}

The main goal of this paper is to present a detailed analysis of the following problem: Given a beam structure, a level of noise, a certain partial coverage, and a pixelization, how well can we assign a temperature to each pixel?. In other words, how well can we deconvolve the beam to get appropriate temperatures in the pixels?. Here, temperatures are considered to be appropriate when the resulting maps lead to good physical spectra. Hereafter, any beam reversion leading to right spectra is referred to as a "S-deconvolution". In the absence of noise, the possibility of performing a good S-deconvolution essentially depends on the ratio between the beam area and that of the chosen pixel. In practice, S-deconvolution is not feasible for too large values of this ratio; in other words, if we fix the beam, $\mathrm{S}$-deconvolution is not feasible for too small pixel sizes. For a given beam and a certain mathematical method, there is a minimum pixel size allowing S-deconvolution. For values smaller than this minimum, too many pixels can be placed inside the beam and $\mathrm{S}$-deconvolution is not possible. The minimum size corresponding to two S-deconvolution methods has been estimated in various cases. Both methods lead to similar minimum values of

the pixel size around $\theta_{F W H M} / 2$. These values depend on the level of the uncorrelated noise produced by the instruments.

It is worthwhile to emphasize that we are not interested in assigning temperatures to hundreds of pixels located inside the beam. This assignation can be useful in other contexts; however, in our case, the important point is that the spectra contained in 
the S-deconvolved maps must be similar to the true physical spectra (up to the scales corresponding to the pixel size). Unfortunately, this type of deconvolution requires a moderate number of pixels inside the beam. This number will be estimated below in various cases.

From $\S 2$ to $\S 4$, pixelization is considered in the framework of pure beam reversion, without analyzing particular experiments; however, in $\S 5$, we focus our attention on PLANCK multifrequency observations and, then, pixelization is discussed taking into account both previous conclusions about beam reversion and some physical constraints due to difraction, observational strategy, et cetera.

In Sáez, Holtmann \& Smoot (1996) and Sáez \& Arnau (1997), the modified power spectrum

$$
E_{\ell}(\sigma)=\frac{32 \pi^{3}}{(2 \ell+1)^{2}} \int_{\alpha_{\min }}^{\alpha_{\max }} C_{\sigma}(\alpha) P_{\ell}(\cos \alpha) \sin \alpha d \alpha
$$

was described. Functions $P_{\ell}$ are the Legendre polinomial normalized as follows: $\int P_{\ell} P_{\ell^{\prime}} d(\cos \theta)=\left[(2 \ell+1) / 8 \pi^{2}\right] \delta_{\ell \ell^{\prime}}$. As explained in those papers, this type of spectrum can be easily found from both theory and maps. Comparisons of the modified spectra obtained from theory with those extracted from simulated or observational maps are appropriate to take into account pixelization, partial coverage and beam features, simultaneously. Sometimes, the estimation and use of the well known $C_{\ell}$ coefficients -although possible - is not the best procedure. In Eq. (1), the effect of pixelization is simulated by the angle $\alpha_{m i n}$, which is the angle separating two neighbouring nodes, while the angle $\alpha_{\max }$ depends on the area of the covered region; this angle is to be 
experimentally obtained (Sáez \& Arnau, 1997), it is smaller than the size of the map and large enough to include as much scales as possible. The autocorrelation function $C_{\sigma}(\alpha)$ is

$$
C_{\sigma}(\alpha)=\left\langle\left(\frac{\delta T}{T}\right)_{\sigma}\left(\vec{n}_{1}\right)\left(\frac{\delta T}{T}\right)_{\sigma}\left(\vec{n}_{2}\right)\right\rangle,
$$

where $\alpha$ is the angle formed by the unit vectors $\vec{n}_{1}$ and $\vec{n}_{2}$, the angular brackets stand for an average over many full realizations of the CMB sky and, quantity $(\delta T / T)_{\sigma}(\vec{n})$ is the temperature contrast in the direction $\vec{n}$ after smoothing with a Gaussian beam having a certain $\sigma=0.425 \theta_{F W H M}$. The modified spectra are used below to analyze some simulated maps.

\section{SIMULATIONS}

The angular power spectrum $C_{\ell}=\sum_{m=-\ell}^{m=\ell}\left|a_{\ell m}\right|^{2} /(2 \ell+1)$ is only an auxiliary element in our estimations. We are not particularly interested in any choice and, consequently, we have used the same spectrum as in Sáez, Holtmann \& Smoot (1996). It corresponds to the minimum cold dark matter model with a baryonic density parameter $\Omega_{B}=0.03$ and a reduced Hubble constant $h=0.5$. The $C_{\ell}$ coefficients have been taken from Sugiyama (1995) and renormalized according to the four-year COBE data $\left(Q_{r m s_{P} S} \simeq\right.$ $18 \mu K$, Gorski et al. 1996). Our simulations are performed by using the Fast Fourier Transform (see Sáez, Holtmann \& Smoot 1996 and Bond \& Efstathiou 1987) and, then, a certain beam is used to average temperatures; thus, we obtain maps which must be deconvolved with the same beam. After S-deconvolution, the resulting map 
must be compared with the initial one.

\section{IDEAL BEAM S-DECONVOLUTION}

Two methods are proposed to perform a S-deconvolution of the beam in the absence of noise (ideal case). The efficiency of these methods is verified and the limits for their application are discussed. For appropriate coverages and beam structures, the size of the smallest pixels compatible with beam reversion is estimated in each case. The conclusions obtained in this section are important to understand realistic $\mathrm{S}_{-}$ deconvolution in noisy maps $(\S 4)$.

\subsection{BEAM}

We begin with a Gaussian spherically symmetric beam. If the direction of the beam center is $\vec{n}$, the measured temperature $T(\vec{n})$ is given by the following average:

$$
T(\vec{n})=\frac{1}{2 \pi \sigma^{2}} \int T^{*}(\theta, \phi) e^{-\left[\theta^{*}(\vec{n})\right]^{2} / 2 \sigma^{2}} \sin \theta d \theta d \phi
$$

where $\sigma$ defines the beam size, the angles $\theta$ and $\phi$ are the spherical coordinates of a certain pixel, the element of solid angle is $d \Omega=\sin \theta d \theta d \phi$ and, quantity $\theta^{*}$ is the angle formed by the direction $(\theta, \phi)$ and the observation direction $\vec{n}$.

Small pixels can be considered as surface elements and, consequently, Eq. (3) can be discretized as follows:

$$
T(\vec{n})=\frac{1}{2 \pi \sigma^{2}} \sum_{i} T_{i}^{*} e^{-\left(\theta_{i}^{*}\right)^{2} / 2 \sigma^{2}} \frac{d S_{i}^{2}}{R^{2}}
$$


where the subscript $i$ stands for the $i$-th pixel; here, $T_{i}^{*}$ and $d S_{i}$ are the temperature and the area of the $i$-th pixel, respectively, and $\theta_{i}^{*}$ is the angle formed by this pixel and the beam center. The exponential tends rapidly to zero as $\theta_{i}^{*}$ increases beyond $\sigma$; hence, only a small number of pixels are significant in order to estimate $T(\vec{n})$. Furthermore, due to technical reasons, the beam could receive energy from a reduced number of pixels (not from all the significant pixels in an ideal infinite Gaussian beam). By these reasons, we assume that only $q \times q$ pixels are relevant and we give various values to number $q$; these pixels cover a square patch centered at the same point as the beam.

An asymmetric beam of the form

$$
W=\frac{1}{2 \pi \sigma^{2}} e^{\left[a^{2}\left(\theta-\theta^{\prime}\right)^{2}+a^{-2}\left(\phi-\phi^{\prime}\right)^{2}\right] / 2 \sigma^{2}}
$$

has been also considered. The parameter $a$ defines the degree of asymmetry.

Figure 1 illustrates, for $q=7$, three situations corresponding to beams and pixelizations considered below.

\subsection{COVERAGE}

Our choice of an appropriate partial coverage is based on some results obtained in previous papers. In Sáez, Holtmann \& Smoot (1996), it was shown that CMB maps close to $20^{\circ} \times 20^{\circ}$ can be simulated -with good accuracy- neglecting curvature and using the Fourier transform. The effects of partial coverage were analyzed in detail

in Sáez \& Arnau (1997), these authors proved that, although a $20^{\circ} \times 20^{\circ}$ map does 
not suffice to get the angular power spectrum, a few tens of $20^{\circ} \times 20^{\circ}$ maps allow us to find the power spectrum with a high accuracy for $\ell>200$. On account of this previous result, forty $20^{\circ} \times 20^{\circ}$ maps are used in this paper to get each angular power spectrum. These maps would cover about $40 \%$ of the sky. In the above papers, it was also argued that uncorrelated noise is not expected to be important for coverages greater than or equal to $20^{\circ} \times 20^{\circ}$ and for the noise level expected in modern $\mathrm{CMB}$ experiments. This is true in the sense that the power spectrum can be calculated in the presence of this noise, but the noise can be problematic for $\mathrm{S}$-deconvolution as we will discuss below. These comments point out the interest of considering $20^{\circ} \times 20^{\circ}$ maps and motivate our choice of these regions to begin with our analysis of the S-deconvolution procedure.

\subsection{PIXELIZATION AND EQUATIONS TO BE SOLVED}

In order to compute the integral in (3) using Eq. (41), the area $d S_{i}$ is not required to be independent on $i$; namely, no equal area distributions of pixels are necessary. Furthermore, if we take a large enough number of small pixels covering all the region contributing significantly to the integral (3), moderated variations in the pixel shapes are also admissible. In spite of these comments, equal area and equal shape pixelizations are advantageous -at least from the mathematical point of view- as it is shown along the paper.

Since a $20^{\circ} \times 20^{\circ}$ region is approximately flat, small squares with edges of angular 
lenght $\Delta \theta=\Delta \phi=\Delta$ define an approximately regular pixelization. The number of pixels per edge is $N=20 / \Delta$, where angle $\Delta$ must be given in degrees. Our measurements would cover the pixelized region with a certain strategy. The beam center should point towards each pixel $\alpha$ various times and, then, the mean of the resulting measures could be considered as the smoothed temperature $T_{\alpha}$ corresponding to pixel $\alpha$. From these $T_{\alpha}$ values, a new temperature which does not involve the beam effect, $T_{i}^{*}$, must be assigned to each pixel $i$; namely, the beam must be deconvolved. All along $\S 3$, it is assumed that sistematic errors have been corrected and that the uncorrelated instrument noise is negligible. If $T_{\alpha}$ is the temperature measured by the instrument when the beam center is pointing towards the center of the pixel $\alpha$, according to Eq. (4), we can write

$$
T_{\alpha}=\sum_{i} A_{\alpha i} T_{i}^{*}
$$

with

$$
A_{\alpha i}=\frac{1}{2 \pi \sigma^{2}} e^{-\left(\theta_{i}\left(\vec{n}_{\alpha}\right)^{*}\right)^{2} / 2 \sigma^{2}} \frac{d S_{i}^{2}}{R^{2}},
$$

where $T_{i}^{*}$ is the true temperature in the $\mathrm{i}$-th pixel; namely, the S-deconvolved temperature we are looking for. Similar equations hold for the asymmteric beam (5).

It is evident that the temperatures $T_{i}^{*}$ only define a S-deconvolution if they are an approximate solution of the linear Eqs. (6). Only in this case, the temperatures $T_{i}^{*}$ are similar to the true temperatures averaged by the beam and, consequently, the resulting maps contain the right spectra. This fact strongly restrict the methods for S-deconvolution. Two of them are described in next section. These equations can be 
written in the matrix form $T=A T^{*}$, where $T$ and $T^{*}$ are arrays of $N \times N$ numbers (one number for each pixel) and A is a $N^{2} \times N^{2}$ matrix. The element $A_{\alpha i}$ weights the contribution of the pixel $i$ to the smoothed temperature at pixel $\alpha$. Quantity $A_{\alpha i}$ is assumed to be significant only in the $q \times q$ pixels mentioned in $\S 3.1$.

\subsection{METHODS AND RESULTS}

Two methods are used to estimate the S-deconvolved temperature $T_{i}^{*}$ corresponding to pixel $i$ : In the first one, Eqs. (6) are solved as a linear system of algebraic equations (hereafter LS-deconvolution) where the independent terms are the observed temperatures $T_{\alpha}$. In the second method, equation (3) is considered as a convolution and, then, Fourier transform (FT) and the deconvolution theorem are used to get $T_{i}^{*}$ on the nodes of the 2D Fourier grid (hereafter FTS-deconvolution).

\subsubsection{LS-DECONVOLUTION}

Iterative methods (Golub \& van Loan, 1989; Young, 1971) can be used to solve the system (6). In any of these methods, the matrix $A$ is split as follows $\mathrm{A}=\mathrm{M}-\mathrm{P}$, where $\mathrm{M}$ is any matrix which can be easily inverted. In matrix form, the iteration scheme reads as follows:

$$
T^{*(n+1)}=M^{-1} P T^{*(n)}+M^{-1} T,
$$

where the superscript $n$ stands for the $\mathrm{n}$-th iteration. The necessary and sufficient condition for convergence is that the spectral radius of the matrix $Q=M^{-1} P$ is 
smaller than unity. This radius is the maximum of $\left|\lambda_{i}\right|$, where $\lambda_{i}$ are the eigenvalues of $Q$. Hereafter, the so-called Jacobi method is used. This method corresponds to a particular choice of the matrix $M$. This matrix is assumed to be the matrix formed by the diagonal of $A$, which is denoted $A_{D}$. A sufficient condition for the convergence of the Jacobi method is that matrix $A$ is diagonal dominant $\left(\left|A_{i i}\right|>\sum_{j \neq i}\left|A_{i j}\right|\right.$ for any $i$ ). The dimension of the matrices $A, A_{D}$ and $P$ are $N^{2} \times N^{2}$, where $\mathrm{N}$ is the number of pixels per edge in the map. Since this number is greater than $10^{2}$ in all the practical cases, the dimension of the above matrices is very great and they cannot be stored. Fortunately, this storage is not necessary; in fact, if Eq. (8) is rewritten using indices

$$
A_{i i} T_{i}^{*(n+1)}=T_{i}-\sum_{j<i} A_{i j} T_{j}^{*(n)}-\sum_{j>i} A_{i j} T_{j}^{*(n)}
$$

we see that all the elements of the matrix $A$ necessary to get $T_{i}^{*(n+1)}$ can be obtained when they are necessary, without storage. Of course, a given element can be calculated various times, but no storage is necessary at all.

Equation (8) can be considered as a matrix equation in which each element is a block. Matrix $A$ is split in $N \times N$ blocks and vectors $T$ and $T^{*}$ in $\mathrm{N}$ arrays (blocks) of dimension $N$. Then the resulting $N \times N$ blocks appear to have a q-diagonal structure and diagonal domination gives limit values for quantities $\lambda=e^{-}\left(\Delta^{2} / 2 \sigma^{2}\right)$ and $\theta_{F W H M} / \Delta$. The maximum value of $\lambda$ and the corresponding maximum value of the ratio $\theta_{F W H M} / \Delta$ are given in the first and second columns of Table 1 , respectively, for various $q$ values. The third column gives the ratio $S_{q} / S_{B}$, where $S_{q}$ is the area 
covered by the $q \times q$ significant pixels and $S_{B}$ is the area of the beam (a circle with radius $\left.\theta_{F W H M}\right)$. The top panel of Fig. 1 illustrates the case $q=7$. Eleven pixels are located inside the beam circle (radius $\theta_{F W H M}$ ).

Taking into account that, in general, the condition $\lambda<\lambda_{\max }$ is only a sufficient condition for the convergence of the Jacobi method (not necessary), we have studied numerically many cases in which $\lambda \geq \lambda_{\max }$ with the hope of getting new convergent cases. The result is that the Jacobi method has never converged for $\lambda>\lambda_{\max }$. This result points out that, in practice, the condition $\lambda \leq \lambda_{\max }$ is also necessary for LS-deconvolution. This information suffices for us. A rigorous mathematical study about the necessary character of this condition is not appropriate here. From the intuitive point of view, the existence -in practice- of a $\lambda_{\max }$ value is an expected result, in fact, in the absence of a $\lambda_{\max }$, it would be possible to assign deconvolved temperatures to billions (an arbitrary number) of small pixels placed inside the beam and, furthermore, the right spectrum could be recovered up to the spatial scales of these small pixels; this would be a nonsense.

When LS-deconvolution applies, it is an accurate method. In fact, for $\theta_{F W H M}=$ $8.8^{\prime}$ and $\Delta=4.6875^{\prime}$, about 24 iterations suffice to get a very good deconvolved map. After these iterations, the relation $\left[\sum_{i=1}^{N^{2}}\left(T_{i}^{(n+1)}-T_{i}^{(n)}\right)^{2}\right]^{1 / 2} / N^{2}<10^{-7}$ is satisfied and, consequently, the method is converging towards a certain map. The question is: are the numerical iterations converging to a good S-deconvolved map with the right spectrum? In order to answer this question, we proceed as follows: (1) the Fast 
Fourier Transform (FFT) is used to do a simulation $\left(S_{1}\right)$ which does not involve either beam or noise, $(2)$ a second map $\left(S_{2}\right)$ is obtained -from $S_{1}$ - by using a certain beam for smoothing, (3) the map $S_{2}$ is deconvolved using the Jacobi method to get a new map $\left(S_{3}\right),(4)$ the three above steps are repeated forty times (see $\left.\S 3.2\right)$ and, (5) the method described in the introduction (see also Sáez, Holtmann \& Smoot 1996 and Sáez \& Arnau 1997) is used to obtain the modified spectrum in three cases: before smoothing (from $S_{1}$ maps), after smoothing (from $S_{2}$ maps) and, after deconvolution (from $S_{3}$ maps), these spectra are hereafter referred to as $E_{1 \ell}, E_{2 \ell}$, and $E_{3 \ell}$, respectively. If deconvolution is a good enough $\mathrm{S}$-deconvolution, spectrum $E_{3 \ell}$ should be comparable with $E_{1 \ell}$. Whatever the deconvolution method may be, these five steps allow us to analize the resulting deconvolved maps. In all the Figures of this paper which show the three above spectra, pointed, dashed, and solid lines correspond to $E_{1 \ell}, E_{2 \ell}$, and $E_{3 \ell}$, respectively. The top right panel of Fig. 2 shows the resulting spectra for the LS-deconvolution under consideration $\left(\theta_{F W H M}=8.8^{\prime}\right.$ and $\left.\Delta=4.6875^{\prime}\right)$. We see that the dotted line $\left(E_{1 \ell}\right)$ is almost indistinguishable from the solid one $\left(E_{3 \ell}\right)$ for $\ell<2000$. This result qualitatively proves the goodness of the iterative LS-deconvolution. In order to compare the spectra $E_{1 \ell}$ and $E_{3 \ell}$ quantitatively, the following quantities are calculated and presented in Table 2 (entries 7 and 8): The mean, $M 1$, of the quantities $\ell(\ell+1) E_{\ell} \times 10^{10}$ corresponding to the spectrum $E_{1 \ell}$ (col. [3]), the mean, $M 2$, of the differences $E_{1 \ell}-E_{3 \ell}$ (col. [4]), the mean MA of $\left|E_{1 \ell}-E_{3 \ell}\right|$ (col. [5]), and the typical deviation, $\Sigma$, of the differences of column (4) (col. [6]). The above quantities are 
computed in appropriate $\ell$-intervals (col. [2]). Entries 7 and 8 show values of $|M 2|$, $M A$ and $\Sigma$ much smaller than $|M 1|$, which means that the spectra $E_{1 \ell}$ and $E_{3 \ell}$ are very similar in both $\ell$-intervals $(40,1000)$ and $(1000,2000)$. It is also remarkable that $|M 2|$ is much smaller than MA, which means that spectrum $E_{3 \ell}$ oscillates around spectrum $E_{1 \ell}$ giving positive and negative values of $E_{1 \ell}-E_{3 \ell}$ which cancel among them.

Table 2 compares other pairs of spectra displayed in Figs. 2 and 3. Column (1) gives the Figure and panel where each pair of spectra are displayed. Tables 3 and 4 have the same structure as Table 2, but they compare pairs of spectra contained in Figs 4 and 5, respectively. The interpretation of the data exhibited in these Tables is straightforward. For a given entry, the compared spectra are similar if the quantities $|M 2|, M A$ and $\Sigma$ are much smaller than $|M 1|$. Given two entries with similar $|M 1|$ values, the smaller the values of $|M 2|, M A$ and $\Sigma$, the greater the similarity between the spectra (the better the deconvolution if we are comparing $E_{1 \ell}$ and $E_{3 \ell}$ spectra).

\subsubsection{FTS-DECONVOLUTION}

The method based on the FT only can be used in the case of small enough coverages (almost flat regions) allowing a uniform pixelization. The region covered by the observations should be a square with no much more than $\sim 20^{\circ}$ per edge; thus, curvature can be neglected and the covered area can be considered as a square where the angles $\theta$ and $\phi$ play the role of cartesian coordinates. Equation (循) can be then 
seen as a convolution of the function $U(\theta, \phi)=T^{*}(\theta, \phi) \sin (\theta)$ with the Gaussian beam function $W=\frac{1}{2 \pi \sigma^{2}} e^{\left[\left(\theta-\theta^{\prime}\right)^{2}+\left(\phi-\phi^{\prime}\right)^{2}\right] / 2 \sigma^{2}}$, where coordinates $\theta^{\prime}$ and $\phi^{\prime}$ define the observation direction $\vec{n}$. Then, the deconvolution theorem ensures that the Fourier transform of function $U$ is

$$
U(\vec{k})=T(\vec{k}) / W(\vec{k})
$$

where $\vec{k}$ is a vector in the 2-dimensional Fourier space. Given a smoothed map and a window function, we can find their Fourier transforms $T(\vec{k})$ and $W(\vec{k})$ and, then, Eq. (10) plus an inverse FT allows us to find the deconvolved map. Unfortunately, the use of the FT is not compatible with spherically assymmetric rotating beams. If one of these beams measures in such a way that its orientation changes from measure to measure, Eq. (3) is not a convolution anymore and, consequently, the FTS-deconvolution does not apply; hence, the FT can be used either in the case of a spherically symmetric beam or in the case of a nonspherical nonrotating beam which measures preserving its orientation.

The maximum value of the ratio $\theta_{F W H M} / \Delta$ compatible with FTS-deconvolution has been derived using simulations. In all the $20^{\circ} \times 20^{\circ}$ simulations, we have taken $N=256\left(\Delta=4.6875^{\prime}\right)$, while quantity $\theta_{F W H M}$ has been varied appropriately. The code for FTS-deconvolution has been run in each case. This code follows the five steps of the process described above for analyzing deconvolved maps. The left panels of Fig. 2 show the spectra $E_{1 \ell}, E_{2 \ell}$, and $E_{3 \ell}$, for different values of $\theta_{F W H M}$. The top left panel, which corresponds to $\theta_{F W H M}=10^{\prime}$, shows that the spectra $E_{1 \ell}$ and $E_{3 \ell}$ 
are very similar in all the $\ell$-interval $(2,2000)$. For $\theta_{F W H M} \sim 11^{\prime}$ (middle left panel), these spectra are very similar for $2 \leq \ell \leq 1000$, while they become a little diferent in the interval $(1000,2000)$. For $\theta_{F W H M}>11^{\prime}$, the differences between $E_{1 \ell}$ and $E_{3 \ell}$ grow rapidly and, for $\theta_{F W H M}=11.5^{\prime}$ (bottom left panel), these spectra are very different. This is quantitatively confirmed by the numbers presented in entries 1 to 6 of Table 2, where we see that, in the interval $(1000,2000)$, quantities $|M 1|, M A$ and $\Sigma$ increase as $\theta_{F W H M}$ does. The maximum value of $\theta_{F W H M} / \Delta$ appears to be $\sim 2.3$. This means that, in the case $1.87<\theta_{F W H M} / \Delta \leq 2.3$, the FTS-deconvolution applies and the LSdeconvolution does not. The most dense grid compatible with FTS-deconvolution is shown in the middle panel of Fig. 1. In this case, around 16 pixels can be placed inside the beam circle.

An asymmetric nonrotating beam of the form (5) has been also deconvolved for various values of the parameters $a$ and $\theta_{F W H M}$. The middle right panel of Fig. 2 shows the results of the FTS-deconvolution for $a=1.29$ and $\theta_{F W H M}=7.75^{\prime}$. These results are good in the full $\ell$-interval $(2,2000)$ (see entries 9 and 10 of Table 2). This choice of the parameters $a$ and $\sigma$ simulates an asymmetric beam whose effective $\theta_{F W H M}$ along the $\theta$-axis $(\phi$-axis $)$ is $\theta_{1}^{e f f}=\theta_{F W H M} / a \simeq 6^{\prime}\left(\theta_{2}^{e f f}=a \theta_{F W H M} \simeq 10^{\prime}\right)$. This beam and the most dense pixelization allowing its FTS-deconvolution are shown in the bottom panel of Fig. 1. Around nine pixels are located inside the beam ellipse. Other $a$ and $\sigma$ values have been also considered to conclude that FTS-deconvolution is possible when both $\sigma_{1}^{\text {eff }} / \Delta$ and $\sigma_{2}^{\text {eff }} / \Delta$ are smaller than $\sim 2.3$ (this constraint is equivalent to that 
obtained in the spherically symmetric case). Finally, we have compared the effect of a symmetric beam with $\theta_{F W H M}=10^{\prime}$ and that of the asymmetric nonrotating beam described above. In order to do this comparison we have obtained forty $S 2$ maps with each beam. The spectra obtained from these S2 maps are displayed in the bottom right panel of Fig. 2 and quantitatively compared in entries 11 and 12 of Table 2. The solid (pointed) line corresponds to the asymmetric (symmetric) beam. Results show that the deformations of the original spectrum produced by these beams are different; namely, that the $E_{2 \ell}$ spectra are distinct (significant asymmetry).

Vanishing instrumental noise has been assumed so far; nevertheles, partial coverage introduces a kind of sky noise (an uncertainty). In order to estimate this noise for a coverage of forty $20^{\circ} \times 20^{\circ}$ maps, we compare the $S 1$ spectrum extracted from these maps with the theoretical spectrum (which would correspond to many realizations of the full sky). Both spectra are presented in Fig. 3, where the solid (pointed) line corresponds to the S1 (theoretical) spectrum. The quantitative comparison of these spectra is given in entries 13 and 14 of Table 2 . We see that the deviations with respect to the true spectrum -produced by the partial coverage under consideration- are greater than those produced by good S-deconvolutions (compare entries 13 and 14 of Table 2 with the pairs of entries $1-2,7-8$, and $9-10$. Compare also the corresponding panels in the Figures). The deviations decrease as the coverage increases. 


\section{BEAM DECONVOLUTION IN NOISY MAPS}

In $\S 3$, negligible uncorrelated noise has been assumed, thus, for a given beam, a minimum pixel size for LS-deconvolution and another one for FTS-deconvolution have been found. These minima define theoretical restrictions for admissible pixelization; nevertheless, in the presence of uncorrelated noise, stronger restrictions on pixel sizes could appear and, consequently, S-deconvolution could be impossible for some sizes close to the minimum size obtained in the absence of noise. Which is the minimum size allowing FTS-deconvolution in the presence of a certain level of uncorrelated noise? We are going to study this question.

As it is well known, the amount of noise in a map depends on the observing time per pixel, $t_{p i x}$, which is inversely proportional to the pixel area. At pixel i, the noise contributes to the temperature an amount $\delta T_{i}^{N}$. It is assumed that the noise is uncorrelated and has uniform variance $\sigma_{N}^{2}$; i.e., $\left\langle\delta T_{i}^{N} \delta T_{j}^{N}\right\rangle=\sigma_{N}^{2} \delta_{i j}$. The relation $\sigma_{N}=s /\left(t_{p i x}\right)^{1 / 2}$ can be used to estimate the level of uncorrelated noise in the pixelized map, where $s$ is the detector sensitivity (see Knox, 1995). For $s=$

$200 \mu K \sqrt{(\mathrm{sec})}, \Delta=4.6875^{\prime}$, and a year of uniform full-sky coverage, the level of noise is $\sigma_{N} \simeq 93.9 \mu K$. Furthermore, using the $C_{\ell}$ numbers of $\S 2$ with $\Delta=4.6875^{\prime}$ and $\theta_{F W H M}=8.8^{\prime}$, the expected signal $S=\left[(1 / 4 \pi)\left(\sum_{\ell}(2 \ell+1) C_{\ell} e^{-\sigma^{2} \ell^{2}}\right)\right]^{1 / 2}$ takes on the value $S \simeq 108 \mu K$. Therefore, for the above choice of $s, \theta_{F W H M}$, and $\Delta$, the signal to noise ratio $S / \sigma_{N}$ is close to 1 and, consequently, noise cannot be neglected a priori in order to do beam S-deconvolution. In other realistic cases, the situation 
is similar. Since $\sigma_{N}$ is proportional to $\left(t_{p i x}\right)^{-1 / 2}$, too long observation times would be necessary to rise $S / \sigma_{N}$ significantly. Fortunately, technological progress leads to smaller and smaller $s$ values and, accordingly, the ratio $S / \sigma_{N}$ increases. In the PLANCK project of the European Spatial Agency there are two instruments: the Low Frequency Instrument (using radiometers) and the High Frequency Instrument (using bolometers). One of the radiometers $\left(\nu=100 G H z\right.$ and $\left.\theta_{F W H M}=10^{\prime}\right)$, working at $20^{\circ} K$, would produce a noise $\sigma_{N} \sim 54 \mu K$ (for pixels with $\Delta=4.6875^{\prime}$ ) during a year of uniform coverage; moreover, for one of the bolometers $(\nu=143 G H z$ and $\left.\theta_{F W H M}=10.3^{\prime}\right)$, which would work at $\sim 0.1^{\circ} K$, the noise would be $\sigma_{N} \sim 16.4 \mu K$ for the same pixels and time coverage. These data are taken into account below in order to analyze the perspectives of beam S-deconvolution in the framework of the most accurate project for anisotropy detection in small angular scales (the PLANCK mission of the European Spatial Agency).

In spite of the fact that LS-deconvolution has been very useful in order to analyze and understand the existence of a minimum size for pixelization, in practice, only the FTS-deconvolution has been used -so far- in the noisy case. The maximum value of $\theta_{F W H M} / \Delta$ compatible with FTS-deconvolution depends on the level of uncorrelated noise. For this type of noise, if the average in Eq. (2) is performed on many sky realizations, the resulting $C_{\sigma}(\alpha)$ values must be very small; nevertheless, if the average is done in a $20^{\circ} \times 20^{\circ}$ patch, the $C_{\sigma}(\alpha)$ values can be relevant, which means that, on the patch, the noise is not properly uncorrelated (its spectrum is unknown). This 
fact is important in order to understand the effect of this noise on FTS-deconvolution of $20^{\circ} \times 20^{\circ}$ maps. In the presence of a certain noise which is independent on the signal, FTS-deconvolution could be performed using the so-called optimal Wiener filter (Press et al. 1988). In such a case, the spectrum of the function $U$-defined above- is estimated as follows:

$$
U(\vec{k})=T(\vec{k}) \Phi(\vec{k}) / W(\vec{k})
$$

where

$$
\Phi(\vec{k})=1-\frac{|N(\vec{k})|^{2}}{|U(\vec{k})|^{2}+|N(\vec{k})|^{2}} .
$$

In the absence of noise, function $\Phi$ takes on the form $\Phi(\vec{k})=1$ and Eq. (11) reduces to Eq. (10). Equations (11) and (12) cannot be used in practice to deconvolve the beam (unknown spectrum of a given $20^{\circ} \times 20^{\circ}$ noise realization); nevertheless, these equations are useful to understand why the noise can be neglected in some cases. The maximum of the $|U(\vec{k})|^{2}$ values corresponding to forty $20^{\circ} \times 20^{\circ}$ simulations -based on the model of $\S 2$ - has been estimated to be 1.7 , while the maximum of $|N(\vec{k})|^{2}$ obtained from the same number of simulations of pure noise (uncorrelated in great regions) has appeared to be proportional to the level of noise $\sigma_{N}$. For $\sigma_{N}=16.4 \mu K$, the resulting maximum is $2.7 \times 10^{-3}$. In this case -and also for any current or planned experimentthe amplitude corresponding to $|N(\vec{k})|^{2}$ is much smaller than that of $|U(\vec{k})|^{2}$. This smallness -relative to that of the signal- indicates that, in realistic noisy cases, the filter function is close to $\Phi(\vec{k})=1$ and the following question arises: Is it possible to take $\Phi(\vec{k})=1$ (noise neglection) to reverse beam smoothing? No theoretical 
arguments have been found to answer this question. Numerical simulations have been necessary. Results obtained from simulations are displayed in Fig. 4. In all the cases studied, the noise has been neglected and Eq. (10) has been used to perform FTS-deconvolution. Good results indicate that noise neglection is appropriate.

In the left panels of Fig. 4 , the level of noise is $16.4 \mu \mathrm{K}$. For $\theta_{F W H M}=8.8^{\prime}$ (top left panel) spectra $E_{1 \ell}$ and $E_{3 \ell}$ are quasi indistinguishable in the $\ell$-interval $(2,2000)$. For $\theta_{F W H M} \sim 9.5^{\prime}$, a small difference between these spectra appears for $1000 \leq \ell \leq 2000$ (middle left panel). Finally, $E_{1 \ell}$ and $E_{3 \ell}$ are clearly different for $\theta_{F W H M}=10^{\prime}$ (bottom left panel). Hence, the maximum value of $\theta_{F W H M} / \Delta$ is close to 2 (around 12 pixels inside the beam circle). The right panels of Fig. 4 show the same analysis as the left panels for a noise level of $54.6 \mu \mathrm{K}$. In this case, spectra $E_{1 \ell}$ and $E_{3 \ell}$ are similar in all the interval $(2,2000)$ for $\theta_{F W H M}<8^{\prime}$ (top right panel), small discrepancies in the interval $(1000,2000)$ have already appeared for $\theta_{F W H M} \sim 8.5^{\prime}$ (middle right panel) and, finally, for $\theta_{F W H M} \sim 9^{\prime}$ these discrepancies are important (bottom right panel). For this level of noise, the maximum value of $\theta_{F W H M} / \Delta$ is close to 1.8 (around 10 pixels inside the beam circle). This qualitative analysis of Fig. 4 is confirmed by Table 3 where quantities $M 1, M 2, M A$, and $\Sigma$ are presented in all the cases. 


\section{PIXELIZATION AND DECONVOLUTION IN REALISTIC EXPERIMENTS}

Limitations of the S-deconvolution process have been discussed along the paper. If the pixel size $\Delta$ is taken to be similar to the beam radius $\theta_{F W H M}$, the angular power spectrum can be only estimated for $\ell \leq \ell_{\max }$ with $\ell_{\max } \sim 180 / \Delta \sim 180 / \theta_{F W H M}$; however, for $\Delta \sim \theta_{F W H M} / 2$, the angular power spectrum can be evaluated up to $\ell_{\text {max }} \sim 360 / \theta_{F W H M}$. In these formulae, $\Delta$ and $\theta_{F W H M}$ must be written in degrees. We

see that, for $\theta_{F W H M} \sim 10^{\prime}$, the pixelization $\Delta \sim \theta_{F W H M}\left(\Delta \sim \theta_{F W H M} / 2\right)$ allows us to get the spectrum up to $\ell_{\max } \sim 1080\left(\ell_{\max } \sim 2160\right)$; therefore, for a given beam, the choice of the best feasible pixelization is crucial in order to get maximum information from observations. The minimum pixel size compatible with S-deconvolution (for the methods used in the paper) is hereafter denoted $\Delta_{D E}$.

In realistic experiments, various effects -apart from deconvolution-conditionate the choice of the most appropriate pixelization. In order to discuss these effects, let us focus our attention on PLANCK project (see Tauber, 1999). With a telecope having a diameter $D$, the minimum pixel size allowed by difraction is roughly $\Delta_{D I} \sim 1.22 c / D \nu$, where $c$ is the speed of light and $\nu$ its frequency; hence, this minimum size depends on $\nu$. In a multifrequency experiment, there is a minimum pixel size $\Delta_{D I}$ corresponding to each frequency; for example, in the PLANCK mission $(D=1.5 \mathrm{~m})$, observations will be carried out in nine different frequencies ranging from $30 \mathrm{GHz}$ to $857 \mathrm{GHz}$ 
and, consequently, the size $\Delta_{D I}$ ranges from $30^{\prime}$ to $1^{\prime}$. Furthermore, in the PLANCK case, the line of sight will move on a big circle in the sky each minute; hence, if two successive temperature assignations on the circle are performed at an angular distance $\Delta \alpha$, the time -in seconds- between these asignations is $\Delta t=2.78 \times 10^{-3} \Delta \alpha$. The angle $\Delta \alpha$ must be chosen in such a way that (1) no large overlaping of contiguous beam positions occurs and (2) time $\Delta t$ is greater that the response time of the bolometers. For the chosen period of one minute and $\Delta \alpha \geq 2 \theta_{F W H M}$, there is no overlaping and condition (2) is satisfied for the PLANCK bolometers. A certain pixel size is only admissible if technology plus observational strategy ensure that each pixel is observed a large enough number of times during the mission. Let us estimate this number for PLANCK. For a pixel size $\Delta$, the total number of pixels is $N=1.5 \times 10^{8} \Delta^{-2}$ and admitting uniform coverage during a year (for qualitative estimates) each pixel is observed for a time $\Delta t_{p}=0.2 \Delta^{2} s$; therefore, the number of observations per pixel is $N_{p}=\Delta t_{p} / \Delta t=72 \Delta^{2} / \Delta \alpha$. Finally, for $\Delta \alpha=2 \theta_{F W H M}$, one easily see that the size necessary to obtain $N_{p}$ observations by pixel (during a year of PLANCK mission) is $\Delta^{*}=\frac{1}{6}\left(N_{p} \theta_{F W H M}\right)^{1 / 2}$. Since the $\theta_{F W H M}$ values for PLANCK detectors range from $\sim 30^{\prime}$ to $\sim 4.5^{\prime}$, assuming $N_{p} \geq 100$, we see that $\Delta^{*}$ ranges from $\sim 9.5^{\prime}$ to $\sim 1.3^{\prime}$. This means that, in order to have a number of observations by pixel greater than 100 , the pixel size must be greater than $\Delta^{*}\left(N_{p}=100\right)=\Delta_{100}$.

Given a frequency, there is an optimum pixel size, $\Delta_{O P}$, which will be assumed to be the maximum of the three above sizes $\Delta_{D E}, \Delta_{D I}$ and $\Delta_{100}$. The value of $\Delta_{O P}$ 
depends on the frequency.

In order to separate the foregrounds and the cosmological signal in multifrequency experiments, various frequencies and a unique pixelization must be used and, consequently, the best pixelization would be the maximum of the $\Delta_{O P}$ optimal sizes corresponding to the involved frequencies. This maximum corresponds to the lowest frequency under consideration; for example, in the PLANCK case, if all the frequencies from $30 \mathrm{GHz}$ to $857 \mathrm{GHz}$ are considered, the minimun admissible pixel appears to have a size $\Delta=\Delta_{D I}(\nu=30) \sim 30^{\prime}$, for which, the spectrum can be only estimated up to $\ell_{\max }=360$. Of course, we could consider only the frequencies greater than $53 \mathrm{GHz}$ (with some loss of information) and, then, the minimum pixel is $\Delta=\Delta_{D I}(\nu=53) \sim 17^{\prime}$ and $l_{\max }=630$ and so on.

Let us reconsider the radiometer working at $\nu \sim 100 \mathrm{GHz}$ with $\theta_{F W H M}=10^{\prime}$, which was projected to be inside PLANCK satellite. For this radiometer one easily find $\Delta_{D E} \simeq 5^{\prime}, \Delta_{D I}=8.4^{\prime}$ and $\Delta_{100} \simeq 3^{\prime}$; hence, $\Delta_{O P}=\Delta_{D I}=8.4^{\prime}\left(\ell_{\max } \sim 1290\right)$, for this optimum pixelization, the level of noise is $5.3 \times 10^{-6}$ and the angular spectrum can be obtained for $\ell \leq 1290$ (top panel of Fig. 5 and entries 1 and 2 of Table 4). We now consider the bolometer working at $\nu \sim 143 \mathrm{GHz}$ with $\theta_{F W H M}=10.3^{\prime}$, which was also proposed to measure CMB anisotropy from the PLANCK satellite (phase A study). For this detector we easily find $\Delta_{D E} \simeq 5^{\prime}, \Delta_{D I} \simeq 5.87^{\prime}$ and $\Delta_{100} \simeq 5^{\prime}$; hence, $\Delta_{O P}=\Delta_{D I}=5.87^{\prime}\left(\ell_{\max } \sim 1840\right)$. For the pixelization $\Delta=5.87^{\prime}$ the level of noise is $1.28 \times 10^{-5}$ and we have verified that FTS-deconvolution leads to the right spectrum 
for $\ell \leq 1840$ (bottom panel of Fig. 5 and entries 3 and 4 of Table 4).

For the bolometer working at $857 \mathrm{GHz}$ with $\theta_{F W H M}=4.4^{\prime}$, we get $\Delta_{D I}=1^{\prime}$ and $\Delta_{100} \simeq 1^{\prime}$, but the estimate of $\Delta_{D E}$ is problematic as a result of the high level of noise of this bolometer. Perhaps, in this case, maximum entropy or wavelets could give good results; for example, wavelets could be used to lower the noise before beam deconvolution.

\section{DISCUSSION AND CONCLUSIONS}

We expect that beam deconvolution will be important in order to study some aspects of the observational maps given by experiments as PLANCK. As an example, let us argue that the study of the statistical properties of a given observational map should be performed after deconvolution. In fact, various methods can be used to know if the maps are Gaussian or they obey other statistics; among them, the estimation of the correlation function of pixels where the signal is above a certain threshold (excursion sets, Kaiser 1984) and the local analysis of the spots distributed in the map (Bond and Efstathiou, 1987). Since the beam smoothes the map, it alters the correlations of excursion sets and the structure and distribution of the spots; hence, the above methods for analyzing statistics should be applied after a good deconvolution.

The separation of the cosmic signal and the foregrounds requires a unique appropriate pixelization. In the PLANCK case, we have seen that the optimal size for this pixelization, $\Delta_{O P}$, coincides with the size $\Delta_{D I} \sim \theta_{F W H M}$ corresponding to the lowest 
frequency under consideration; nevertheless, other studies can be imagined (statistical analysis et cetera) which could be performed on the maps corresponding to a given frequency (without previous separation).

For small enough (but feasible) values of $\sigma_{N}$ plus a certain beam (either spherical with a $\theta_{F W H M}$ or asymmteric), our codes allow us to find the most appropriate pixel size for deconvolution $\Delta=\Delta_{D E}$. For the corresponding pixelization, FTSdeconvolution leads to a good estimation of the angular power spectrum in the most wide $\ell$-interval. The size $\Delta_{D E}$ must be compared to $\Delta_{100}$ and $\Delta_{D I}$ to choose the most appropriate pixelization $\Delta=\Delta_{O P}$. In the case $\Delta_{O P}>\Delta_{D E}$, the study about beam reversion presented in $\S 3$ to $\S 5$ proves that S-deconvolution can be performed using very simple methods. For levels of noise much higher that those of previous sections, further study is necessary; maximum entropy, wavelets or other methods should be tried out.

The goodness of a certain pixelization against beam S-deconvolution has appeared to be weakly dependent on the particular mathematical method used to reverse the beam average. This fact suggests that deconvolution procedures different from those of this paper could alter its results. Altough this suggestion should be a motivation for studying new methods to get approximate solutions of Eqs. (6) (S-deconvolutions), the structure of the system of linear equations to be solved is always the same and, consequently, all the mathematical methods could exhibit similar limitations to solve it. Indeed, we believe that new deconvolution methods could lead to some modifica- 
tions of the results of this paper, but not to very different values of $\Delta_{D E}$.

Our estimates show that, in the absence of any problem with beam asymmetry, current technology could lead to a good estimate of the angular power spectrum (of the total signal including foregrounds) in a $\ell$-interval which depends on frequency. In the particular case of two instruments on board of PLANCK, we have used optimum pixelization and forty $20^{\circ} \times 20^{\circ}$ maps to find that the spectra are recovered from $\ell=200$ to $\ell \sim 1300$ in the case of a radiometer and from $\ell=200$ to $\ell \sim 1800$ for a certain bolometer. Since the sky can be divided into $\sim 100$ of these maps and we only need about 40 for a good estimate of the spectrum (for large $\ell$ values), we can select the best forty maps; namely, the maps having minimum contaminations. The uncertainty produced by this partial coverage appears to be a little greater than the errors produced by the implemented deconvolution procedures (this means that these procedures are good enough for us).

The problem with the deviations of the beam structure with respect to spherical symmetry deserves much attention. As discussed above, FTS-deconvolution is compatible with beam asymmetry if the beam orientation is preserved from measurement to measurement. If the experiment is designed in such a way that the beam does not rotate, our codes for FTS-deconvolution work (see $\S 3.4 .2$ and the middle and bottom right panels of Fig. 2); however, if the beam rotates, FTS-deconvolution does not apply and, moreover, operative methods for making beam S-deconvolution are not known; hence, if the assymetry is high enough, beam S-deconvolution is not 
feasible (so far). In short, excepting the case of negligible deviations with respect to spherical symmetry, any effort directed to maintain unaltered the beam orientation during observations seems to be of great interest. Unfortunately, in spatial projects as Planck, the design of the observational strategy does not preserve this orientation.

Given an asymmetric rotating beam, it would be interesting to study the whole effect of asymmetry plus changing orientation. Even if S-deconvolution is not feasible, the estimation of this whole effect could be a further direct application of the techinques used in this paper. The following method seems to be appropriate: (i) average the asymmetric beam -on appropriate shells- to get a new associated one with spherical symmetry, (ii) simulate forty S1 maps, (iii) smooth the S1 maps with the assymmetric beam taking into account the orientation change produced by the observational strategy; thus, we obtain forty S2 maps, (iv) deconvolve the S2 maps with the spherically symmetric beam of reference to get the S3 maps, and (v) estimate the spectra $E_{1 \ell}, E_{2 \ell}$ and, $E_{3 \ell}$. If $E_{1 \ell}$ and $E_{3 \ell}$ are similar enough, the assymetry can be neglected, on the contrary, the differences between these two spectra can be considered as a measure of the whole effect of asymmetry plus rotation. This study should be developed for realistic beams and observational strategies, which is out of the scope of this paper. 
ACKNOWLEDGMENTS. This work has been partially supported by the Spanish DGES (project PB96-0797). Some calculations were carried out on a SGI Origin 2000s at the Centro de Informática de la Universidad de Valencia.

\section{REFERENCES}

Bond, J.R., \& Efstathiou, G., 1987, MNRAS, 226, 655

Golub, G.H., \& van Loan, C.F., 1989, Matrix Computations (Baltimore: Johns Hopkins Press)

Górski, K.M., Banday, A.J., Bennett, C.L., Hinshaw, G., Kogut, A., Smoot, G.F., \&

Wright, E.L. 1996, ApJ, 464, L11

Kaiser, N., 1984, ApJ, 284, L9

Knox, L., 1995, Phys. Rev., 52D, 4307

Press, W.H., Flannery, B.P., Teukolski, S.A., \& Vetterling, W.T., 1988, Numerical

Recipes (New York: Cambridge University press)

Sáez, D., \& Arnau, J.V., 1997, ApJ, 476, 1

Sáez, D., Holtmann, E., \& Smoot, G.F., 1996, ApJ, 473, 1

Young, D.M., 1971, Iterative Solution of Large Linear Systems (New York: Academic Press)

Sugiyama, N., 1995, ApJ Supplement, 100, 281

Tauber, J.A., 1999, Astrophys. Lett. Comm., in press 


\section{FIGURE CAPTIONS}

FIG. 1.- Top (middle) panel shows the relation between the size of a circular beam and that of the pixels for the most dense pixelization compatible with LS (FTS) deconvolution. Bottom panel shows the same for an asymmetric beam and FTSdeconvolution

FIG. 2.- Each panel shows quantity $\ell(\ell+1) E_{\ell} \times 10^{10}$ versus $\log \ell$. No noise is present and $\Delta=4.6875^{\prime}$. All panels, excepting the bottom right one, contain three lines: pointed line gives the spectrum before beam smoothing, dashed line is the spectrum after smoothing and, solid line corresponds to the S-deconvolved spectrum. Left: top, middle and bottom panels correpond to FTS-deconvolutions with beams having $\theta_{F W H M}=10^{\prime}, \theta_{F W H M}=11^{\prime}$, and $\theta_{F W H M}=11.5^{\prime}$, respectively. Right: top (middle) panel shows the same as the left panels for LS-deconvolution and $\theta_{F W H M}=8.8^{\prime}$ (for FTS-deconvolution and the asymmetric beam defined in the text). The dotted (solid) line of the bottom right panel gives the spectrum after smoothing for a spherically symmetric beam with $\sigma=10^{\prime}$ (for the asymmetric beam of the text).

FIG. 3.- The quantities represented are the same as in all the panels of Fig. 2. Solid (pointed) line is the modified spectrum extracted from a coverage of forty $20^{\circ} \times 20^{\circ}$ maps (the theoretical modified spectrum). 
FIG. 4.- The same as in the left panels of Fig. 2. Left: the level of uncorrelated noise per pixel is $\sigma_{N}=16.4 \mu K$. Top, middle and bottom panels correpond to $\theta_{F W H M}=8.8^{\prime}, \theta_{F W H M}=9.5^{\prime}$, and $\theta_{F W H M}=10^{\prime}$, respectively. Right: the same as in left panels for $\sigma_{N}=54.6 \mu K$. Top, middle and bottom panels correpond to $\theta_{F W H M}=8^{\prime}, \theta_{F W H M}=8.5^{\prime}$, and $\theta_{F W H M}=9^{\prime}$, respectively. Pixel size is $\Delta=4.6875^{\prime}$ in all cases.

FIG. 5.- The same as in Fig. 4. Top panel shows the results of FTS-deconvolution for a radiometer of the PLANCK mission described in the text. The pixel size is $\Delta=8.4^{\prime}$. The bottom panel shows the same for a bolometer of PLANCK and $\Delta=5.87^{\prime}$. 
TABLE 1

\section{CONVERGENCE OF THE}

\section{JACOBI METHOD}

\begin{tabular}{cccc}
\hline \hline$q$ & $\lambda_{\max }$ & $\left(\theta_{F W H M} / \Delta\right)_{\max }$ & $S_{q} / S_{B}$ \\
\hline 3 & 0.5000 & 1.998 & 0.72 \\
5 & 0.4565 & 1.879 & 2.25 \\
7 & 0.4559 & 1.877 & 4.43 \\
9. & 0.4559 & 1.877 & 7.32 \\
\hline
\end{tabular}


TABLE 2

COMPARING SPECTRA

FIGS. 2 AND 3

\begin{tabular}{cccccc}
\hline \hline Panel & $\ell$-Interval & $M 1$ & $M 2$ & $M A$ & $\Sigma$ \\
\hline Fig2, Top-Left & $40-1000$ & 13.22 & $4.94 \times 10^{-2}$ & 0.23 & 0.30 \\
Fig2, Top-Left & $1000-2000$ & -14.97 & $-1.25 \times 10^{-2}$ & 0.25 & 0.32 \\
Fig2, Middle-Left & $40-1000$ & 13.14 & -0.12 & 0.27 & 0.35 \\
Fig2, Middle-Left & $1000-2000$ & -14.90 & 0.79 & 0.90 & 1.08 \\
Fig2, Bottom-Left & $40-1000$ & 13.21 & -1.12 & 1.12 & 1.21 \\
Fig2, Bottom-Left & $1000-2000$ & -14.96 & 4.43 & 4.52 & 5.51 \\
Fig2, Top-Right & $40-1000$ & 13.28 & $-5.68 \times 10^{-3}$ & 0.18 & 0.21 \\
Fig2, Top-Right & $1000-2000$ & -15.04 & $8.95 \times 10^{-3}$ & 0.27 & 0.34 \\
Fig2, Middle-Right & $40-1000$ & 13.24 & $8.88 \times 10^{-2}$ & 0.22 & 0.27 \\
Fig2, Middle-Right & $1000-2000$ & -14.99 & $-9.76 \times 10^{-2}$ & 0.36 & 0.45 \\
Fig2, Bottom-Right & $40-1000$ & 9.79 & -3.38 & 3.47 & 4.36 \\
Fig2, Bottom-Right & $1000-2000$ & -11.65 & 3.32 & 3.65 & 4.03 \\
Fig3 & $40-1000$ & 13.48 & -0.35 & 0.61 & 0.76 \\
Fig3 & $1000-2000$ & -14.60 & $7.20 \times 10^{-2}$ & 0.39 & 0.48 \\
\hline
\end{tabular}


TABLE 3

COMPARING SPECTRA

FIG. 4

\begin{tabular}{|c|c|c|c|c|c|}
\hline Panel & $\ell$-Interval & $M 1$ & $M 2$ & $M A$ & $\Sigma$ \\
\hline Top-Left & $40-1000$ & 13.27 & $7.79 \times 10^{-2}$ & 0.20 & 0.23 \\
\hline Top-Left & $1000-2000$ & -15.03 & 0.12 & 0.33 & 0.39 \\
\hline Middle-Left & $40-1000$ & 13.20 & -0.17 & 0.24 & 0.30 \\
\hline Middle-Left & $1000-2000$ & -14.96 & 1.10 & 1.17 & 1.45 \\
\hline Bottom-Left & $40-1000$ & 13.30 & -1.13 & 1.13 & 1.26 \\
\hline Bottom-Left & $1000-2000$ & -15.09 & 4.10 & 4.24 & 5.39 \\
\hline Top-Right & $40-1000$ & 13.26 & 0.16 & 0.26 & 0.31 \\
\hline Top-Right & $1000-2000$ & -15.02 & 0.34 & 0.39 & 0.54 \\
\hline Middle-Right & $40-1000$ & 13.24 & $5.54 \times 10^{-2}$ & 0.22 & 0.29 \\
\hline Middle-Right & $1000-2000$ & -14.99 & 1.24 & 1.28 & 1.47 \\
\hline Bottom-Right & $40-1000$ & 13.27 & -0.43 & 0.51 & 0.64 \\
\hline Bottom-Right & $1000-2000$ & -15.03 & 4.03 & 4.10 & 4.82 \\
\hline
\end{tabular}


TABLE 4

\section{COMPARING SPECTRA}

FIG. 5

\begin{tabular}{cccccc}
\hline \hline Panel & $\ell$-Interval & $M 1$ & $M 2$ & $M A$ & $\Sigma$ \\
\hline Top & $40-700$ & 8.96 & 0.12 & 0.19 & 0.22 \\
Top & $700-1290$ & -14.99 & -0.22 & 0.27 & 0.33 \\
Bottom & $40-1000$ & 9.43 & 0.13 & 0.23 & 0.30 \\
Bottom & $1000-1840$ & -16.24 & 0.31 & 0.40 & 0.49 \\
\hline
\end{tabular}



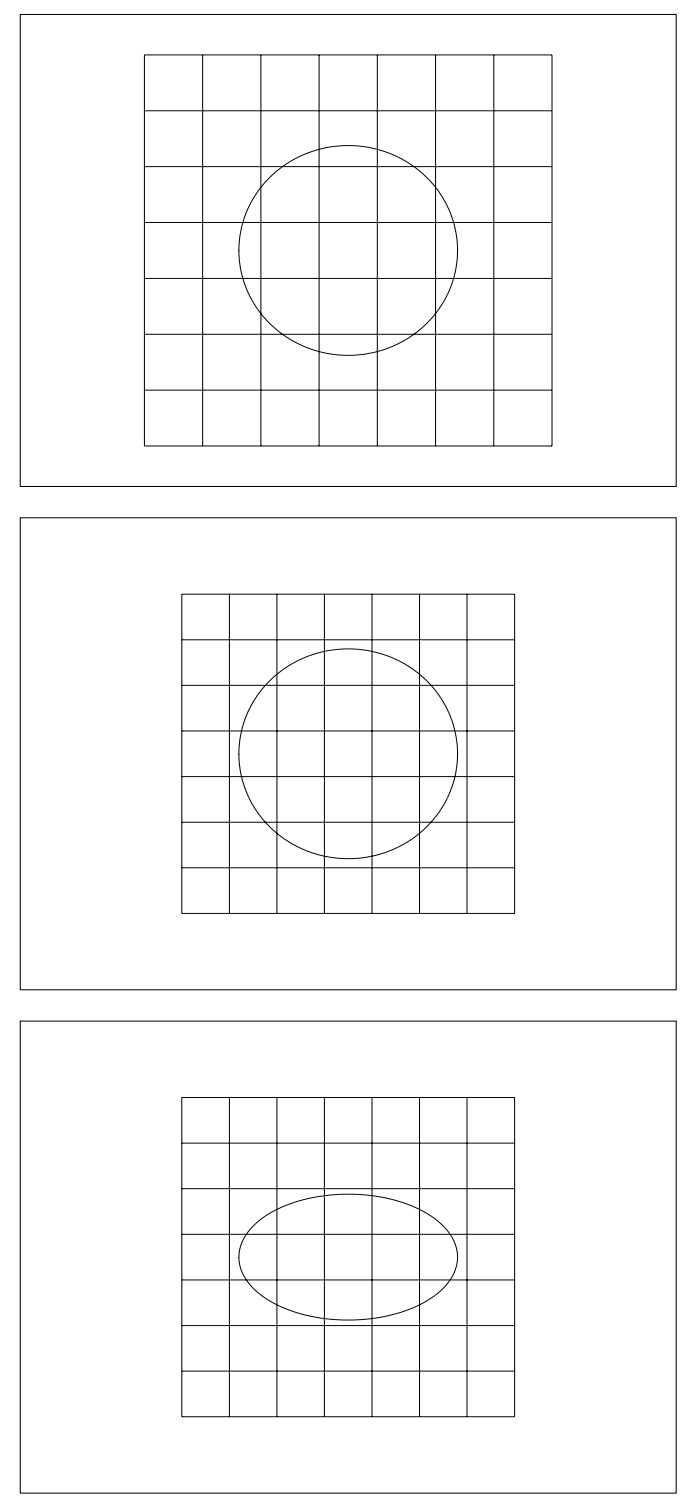




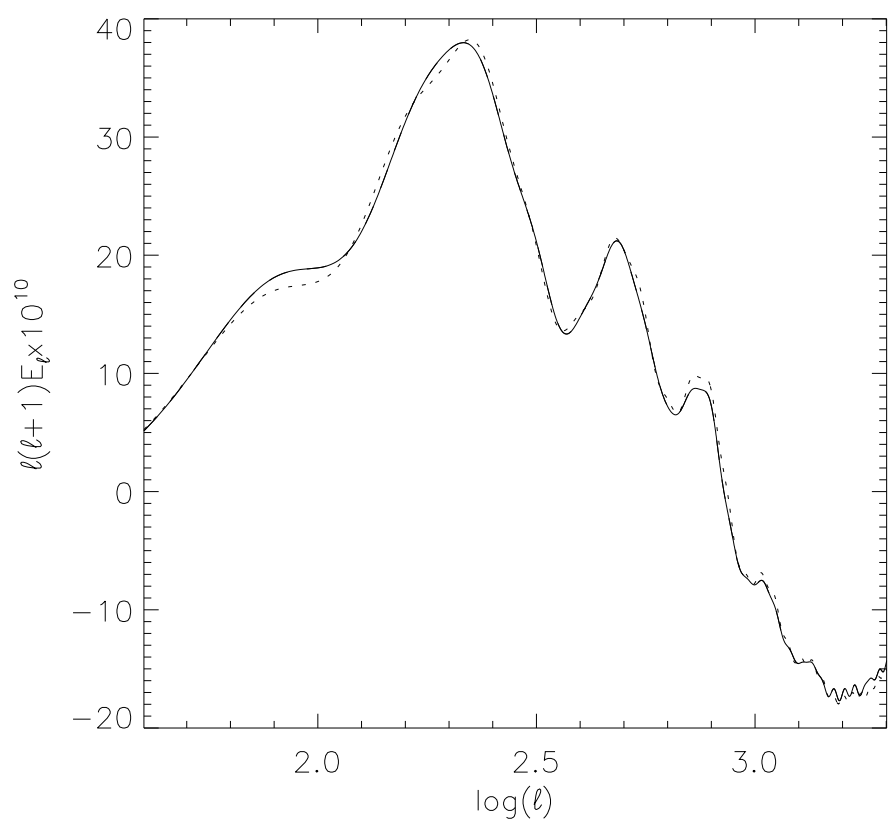



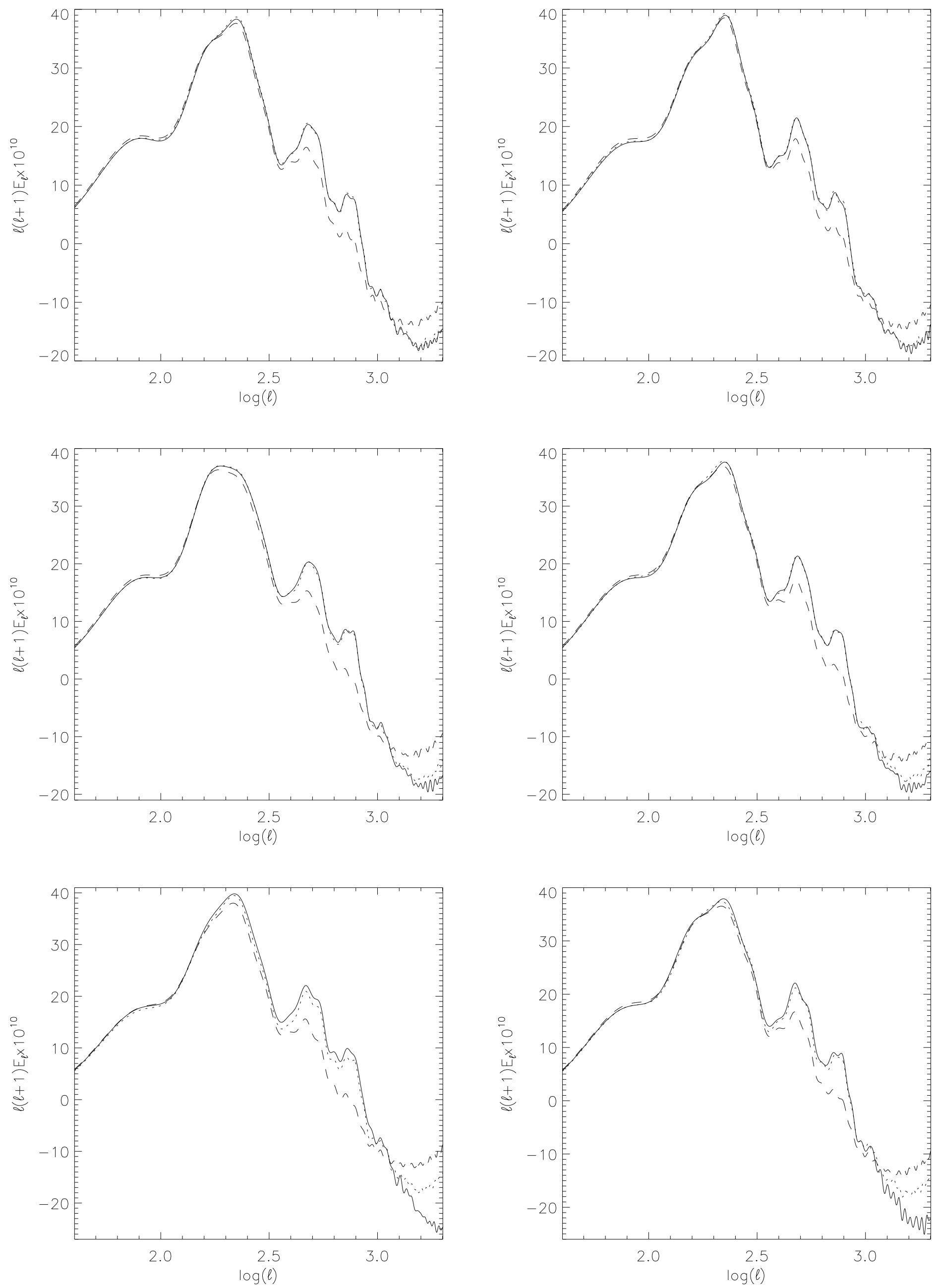

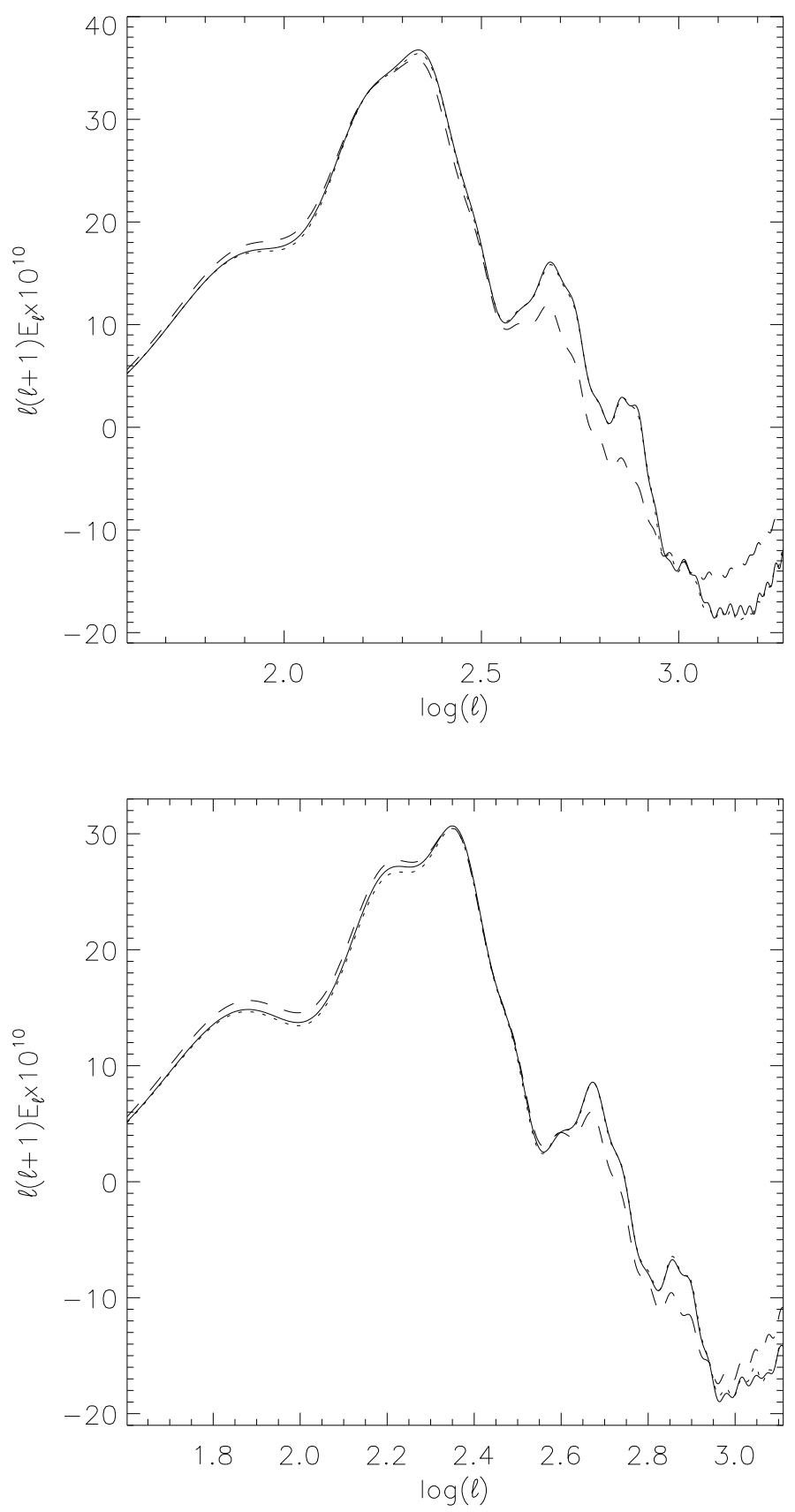